\title{
Ultrafast Dynamical Study of Pyrene- $N, N$-dimethylaniline (PyDMA) as an Organic Molecular Diode in Solid State
}

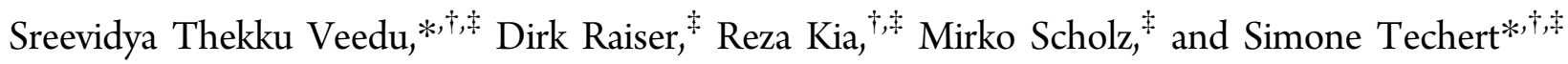 \\ ${ }^{\dagger}$ FS-SCS, Deutsches Elecktronen-Synchrotron (DESY), Notkestr.85, 22607 Hamburg, Germany \\ ${ }^{\ddagger}$ Max Planck Institute for Biophysical Chemistry, Am Fassberg 11, 37077 Göttingen, Germany
}

Supporting Information

ABSTRACT: Femtosecond optical pump-probe spectroscopy has been employed for studying the directly linked electron donor-acceptor system pyrene-N,N-dimethylaniline (PyDMA) in solid state. This DMA-pyrene derivative discussed is being applied as a molecular diode system switching on an ultrafast time scale. Our ultrafast solid-state studies reveal a complex photochemistry of this molecular crystal system. Strong couplings of the optically induced charge-transfer state with the radical ion pair state allow a

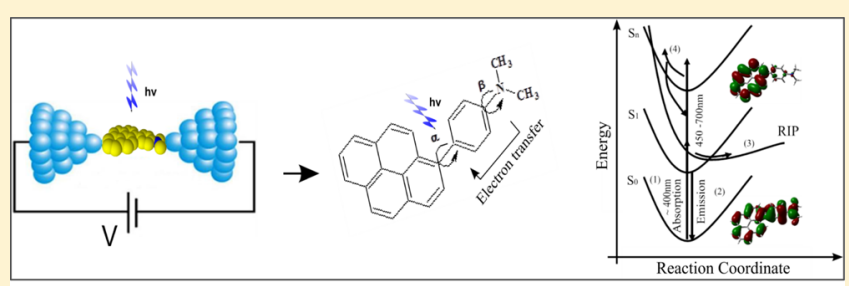

Ultrafast Dynamical Study of PyDMA in solid state femtosecond transition of the latter. One could see on the highest occupied molecular orbital-lowest unoccupied molecular orbital (HOMO-LUMO) description that a pure optical transition switches the system from a conducting to a blocked system because the molecular orbitals (MOs) of DMA moiety lie in a node plane of the LUMO. Within 800 fs the system relaxes back to the ground state and/or forms a radical ion pair, which is the surprising result of our study; when the system was probed further, the system underwent vibrational cooling and enhanced population inversion of the radical ion pair.

\section{INTRODUCTION}

Electron-transfer reactions are fundamental processes in chemistry and also in biology. ${ }^{1-3}$ Light-harvesting complexes are functional centers in plants where sunlight is converted into chemical energy. In this, optical excitation in a chromophore unit leads to the transfer of electrons within the system. However, because of the complexity of the biological photoreaction center, recent spectroscopic efforts have concentrated on a smaller chemical models, which share characteristics with their biological counter parts. A promising model is the so-called D-A (donor-acceptor) systems, which are chemically synthetic molecules with electron-transfer capabilities. The electrical conductivity is a function of the optical state of the system. An optically switching diode is an interesting application of donor-acceptor molecules. ${ }^{4-7}$ Electron donor-acceptor molecules play an inevitable role in the understanding of the charge-transfer process and serve as a testing ground for the coexistent theories of these molecules.

In the past decades, to gain more insight into electrontransfer processes, extensive studies have been carried out on the optical behavior of systems consisting of donor-acceptor groups linked by different bridges. ${ }^{8,9}$ These molecules are also ideal systems for studying the solvation dynamics and also for the demonstration of nonlinear optical properties because of the polarizability of the electrons localized in $\pi$-bonding molecular orbitals. Although the polarizability of a molecule is mainly given by its chemical structure, in particular by the length of the $\pi$-conjugated spacer and the electronic nature of the attached donors and acceptors, ${ }^{10,11}$ it can also be affected by external factors such as strength of radiation, state of matter, and in solution also by the solvent used. In addition, the rate of electron transfer in chemical and biological systems can be sensitively controlled by subtle environmental conditions. ${ }^{12,13}$

Pyrene- $N, N$-dimethylaniline (PyDMA) is a compound in which the electron donor $N, N$-dimethylaniline (DMA) is covalently linked to the electron acceptor pyrene (Scheme 1). Because of the lack of an extended bridge between the donor and acceptor in PyDMA, the physical characteristics of these groups themselves strongly influence the electron-transfer mechanism. This leads to very unusual absorption and emission spectra in the optical regime, and because of this, PyDMA is considered to be a molecular diode where electron donor and electron acceptor moieties are twisted against each other modulating the electron charge-transfer processes. Structurally, for PyDMA (Scheme 1) the two rotational degrees of freedom, rotation around the single bond of pyrene and DMA (rotational angle $\alpha$ ) and rotation of the bond to the amino group in DMA (rotational angle $\beta$ ), can complicate the described electrontransfer reactions of the system.

To characterize molecular diode behavior quantitatively, the framework of Marcus theory ${ }^{14}$ can also be applied. Besides redox changes, the free energy change $\Delta G$ depends also on the inner and outer reorganization energy $\lambda_{\mathrm{i}}$ and $\lambda_{\mathrm{o}}$, respectively. Molecular twisting that changes the orbital overlap and therefore the exchange integral contribute through that channel to the inner energy changes during electron transfer. Because

Received: December 11, 2013

Revised: February 27, 2014

Published: March 6, 2014 
Scheme 1. Molecular Diode (PyDMA)

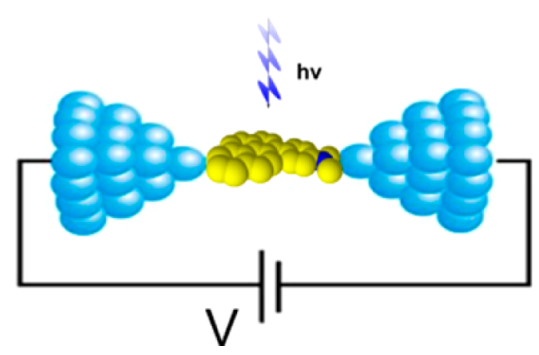

the twist motion belongs to the group of large amplitude motions in molecular structures, various vibrational eigenstates of them are considerably populated at room temperature. So besides orbital overlap, twist motions statistically influence the electron-transfer rates through intramolecular vibrational energy redistribution.

In recent years molecular diodes have attracted much interest because of their strong potential for the use in technological applications. In this type of organic molecules, femtosecond transient absorption spectroscopy in solid state is a very powerful method for determining the mechanisms and kinetics of light conversion into charge. In this paper, we aim for static spectroscopy as well as structure characterization for PyDMA single crystal in its ground state along with computational studies. We will compare the UV-vis spectrum derived from computational simulations with the experimental solid-state spectrum. Furthermore, the ultrafast rate constants of the photoinduced electron transfer have been determined through femtosecond transient absorption spectroscopy. Their time and spectral dependencies will be discussed in the context of the simulations.

\section{EXPERIMENTAL SECTION}

Synthesis and Sample Preparation. Commercially available 1-aminopyrene after diazotization reaction was coupled with $N, N$-dimethylaniline according to a previously reported procedure. ${ }^{15,16}$ The crude product was then purified on an aluminum oxide column with a mixture of cyclohexane/ toluene as eluent under HPLC. The compounds were identified by mass spectrometry and ${ }^{1} \mathrm{H}$ NMR.

For measuring transient absorption, time-correlated singlephoton counting, and X-ray diffraction, single crystals were grown in ethyl acetate and diethyl ether $(2: 1)$ by a slow evaporation technique. The platelike crystals were glued on a glass pin and mounted onto the goniometer head for the experiment.

Crystal Structure Analysis. The crystallographic experiments were carried out at HASYLAB/DESY (DORIS F1 Beamline). The X-ray diffraction data were collected by full sphere routine by $\varphi$ and $\omega$ scan strategy using a twodimensional detector (MarCCD 165) at a wavelength of $0.6 \AA$ at $100 \mathrm{~K}$ with an Oxford Diffraction Cryojet low-temperature cooling device. ${ }^{17}$ The diffraction data were indexed and integrated with the program package XDS. ${ }^{18}$ The crystal structure was solved and refined with the SHELXTL program package. ${ }^{19}$ All the non-hydrogen atoms were refined anisotropically. All of the hydrogen atoms were positioned geometrically in idealized positions and refined with the riding model approximation with isotropic displacement parameters, $U_{\text {iso }}(\mathrm{H})$ $=1.2$ or $1.5 U_{\text {eq }}(\mathrm{C})$. For the molecular graphics the program

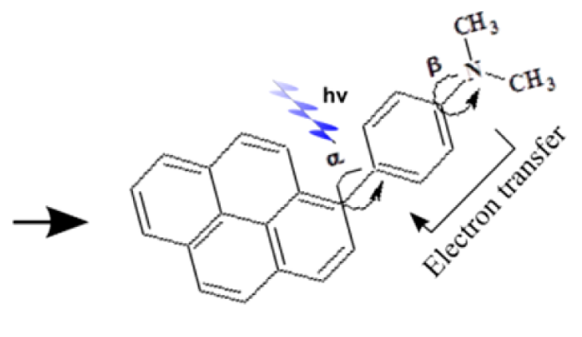

SHELXTL was used. All calculations were carried out using the PLATON software. ${ }^{20}$

Steady-State Absorption Emission Spectral Measurement. The ground-state absorption spectrum was obtained by a UV-vis spectrophotometer (Cary-5E, Varian, Australia), and the steady-state emission spectrum was recorded with a fluorescence spectrometer (Fluorolog 15). ${ }^{21}$

The time-resolved fluorescence decay was obtained by the time-correlated single-photon counting (TCSPC) technique and was analyzed by global analysis using the reference convolution method. ${ }^{22}$

Computational Details. For the ground-state electronic structure calculations density functional theory (DFT) methods with the Becke $^{23}$ three-parameter hybrid functional and LeeYang-Parr's ${ }^{24}$ gradient-corrected correlation functional (B3LYP) was used in the Gaussian03 $(\mathrm{G} 03)^{25}$ program. Basis sets $6-31 G^{*}$ and $6-311++G^{* *}$ were used for molecular orbital calculation and UV-vis spectrum, respectively. The conductorlike polarizable continuum model method (CPCM $)^{26-28}$ with $n$-hexane as solvent was used to calculate the electronic structure and the excited states of the molecule in solution. Time-dependent density functional theory (TD-DFT) ${ }^{29}$ calculations have provided excitation energies of the compound that agree with experimental results. TD-DFT calculation does not provide the electronic structures of the excited states; ${ }^{30}$ however, the electronic distribution and the localization of the singlet excited states may be visualized using electron density difference maps (EDDMs). ${ }^{31}$ GaussSum 2.2 was used for EDDM calculations and for the electronic spectrum simulation. $^{32}$

Transient Absorption Spectroscopic Measurement. The transient absorption measurements were performed with a homemade apparatus using a commercial $\mathrm{Ti}$ sapphire laser system. The apparatus is based on the pump-probe technique where in this case both femtosecond pulses (pump and probe) are generated from the same pulse of the laser system. The laser system that produces the femtosecond pulses consists of a Mira-900F (Coherent Inc.) oscillator, which is pumped by a Verdi V5 and a Hurricane (Spectra Physics) amplifier. The Verdi V5 is a diode-pumped Nd:Yittrium vanadate ( $\left.\mathrm{Nd}: \mathrm{YVO}_{4}\right)$ laser that emits single-frequency green light at a wavelength of $532 \mathrm{~nm}$ due to a frequency-doubling lithium triborate crystal $\left(\mathrm{LBO} / \mathrm{LiB}_{3} \mathrm{O}_{5}\right)$ and has an output power level at $5 \mathrm{~W} .{ }^{33}$ The Mira-900F is a Kerr lens mode-locked oscillator that uses a titanium-doped sapphire $\left(\mathrm{Ti}: \mathrm{Al}_{2} \mathrm{O}_{3}\right)$ as the gain medium as it is commonly used for generation of femtosecond pulses. The generated ultrashort laser pulses have a repetition rate of 76 $\mathrm{MHz}$ and are horizontal polarized. ${ }^{34}$ In this case the tunable wavelength is set to $800 \mathrm{~nm}$. The Hurricane ${ }^{35}$ finally amplifies these ultrashort pulses by using the chirped pulse amplification technique and reduces the repetition rate to $880 \mathrm{~Hz}$. After the 
initial output pulse from the amplifier is split into a pump and a probe pulse, the probe pulse is delayed in time by a translation stage and afterward converted into a white light continuum which covers the complete visible spectrum. In this case the probe pulse is then divided into a reference pulse and a measuring pulse that passes the sample in order to get the optical density (OD). Each pair of pulses is measured twice consecutively (pumped and unpumped) to obtain the change of the optical density $\Delta \mathrm{OD}$, which is measured up to 1000 times for each position of the translation stage. By a stepwise change of the delay time one gains the $\Delta O D$ spectrum as a function of wavelength $\lambda$ and delay time $t$ :

$$
\Delta \mathrm{OD}(\lambda, t)=\mathrm{OD}_{\text {pump-on }}(\lambda, t)-\mathrm{OD}_{\text {pump-off }}(\lambda, t)
$$

To get alternating pump-on and pump-off spectra, every second pump pulse is blocked by a chopper. The wavelength of the pump pulse is set to $400 \mathrm{~nm}$ by generating the second harmonic of the initial pulse using a BBO crystal. The pump and the probe beam are both focused and spatially overlapped in the sample. The pump power is set to approximately $2 \mu \mathrm{J} /$ pulse.

The resulting $\Delta \mathrm{OD}$ spectra were analyzed by fitting the data to a convolution of a multiexponential function for the different decays of transient species with a Gaussian curve representing the temporal shape of the femtosecond pulses.

\section{RESULTS AND DISCUSSION}

The ground-state geometry and the excited-state dynamics of PyDMA were elucidated using a combination of (1) singlecrystal X-ray diffraction, (2) UV-vis absorption spectroscopy, (3) time-dependent DFT calculations, and (4) transient absorption spectroscopy in order to have a precise picture about ground-state geometry from which the dynamics and kinetics of the molecular diode system start.

Crystal Structure. The asymmetric unit comprises one molecule of PyDMA, Figure 1. The compound crystallizes in

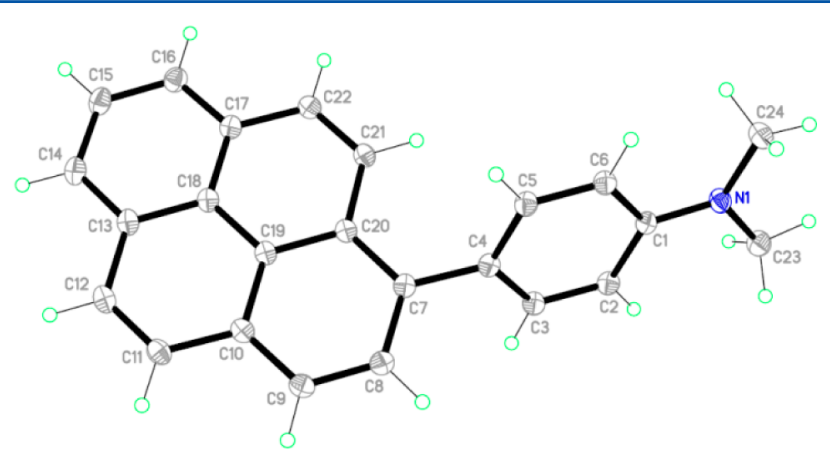

Figure 1. Molecular structure of PyDMA with displacement ellipsoids at $50 \%$ and atom numbering scheme.

the monoclinic system with space group $P 2(1) / c$. The dimethylamine (DMA) group and the phenyl ring are almost coplanar, and they make a dihedral angle of $64.67(3)^{\circ}$ with the pyrene ring. ${ }^{36}$

The crystal packing is stabilized by the intermolecular C$\mathrm{H} \cdots \pi$ interactions, connecting the neighboring molecules into columns along the $c$-axis, which is shown in Figure 2. There is no $\pi-\pi$ interaction because the distance between the centroids is far beyond the limit ( $3.9 \AA$ ) because the DMA and pyrene rings already have a twisted conformation with a dihedral angle of $64.67(3)^{\circ}$. The type of crystal-packing interaction suggests that even in the solid state PyDMA can act on a single molecular diode level. The intermolecular forces are so weak that they do not affect the molecular diode properties. From the crystal structure packing of PyDMA, we can furthermore conclude that the optical properties of solid PyDMA will be comparable to those of PyDMA in solution. The validity of this as well as its consequences for the dynamics of this molecular diode system will be shown in the following sections.

Absorption and Emission Spectroscopy. Figure 3 shows the solid-state absorption and emission spectra of PyDMA. The absorption spectrum shows three maxima at 348, 276, and 240 $\mathrm{nm}$, which are indicative of vibronic progression.

\section{THEORETICAL CALCULATIONS}

Electronic Structure. Because the observed differences in the chemical and physical properties of the system depend mainly on the changes in the ground-state electronic structure, we will discuss in detail the ground-state electronic structure focusing on the frontier orbital components and highest occupied molecular orbital-lowest unoccupied molecular orbital (HOMO-LUMO) energy gap. Some selected frontier molecular orbitals of PyDMA are depicted in Figure 4. The contributions of the most important occupied and virtual as well as the atomic orbital contributions for each of the moieties of the system are listed in Table 1. In the frontier region, neighboring orbitals are often closely spaced, and in such cases, to get a realistic description it is not enough if we consider only the HOMO and LUMO. For this reason, density of states (DOS) diagrams, which incorporate a degree of overlap between the curves convoluted from neighboring energy levels, can give a more representative picture. The density of states of PyDMA plotted as a function of orbital energy is shown in Figure 5, where PyDMA is divided into three segments: the dimethylamino group (DMA), phenyl ring, and pyrene ring.

Each part of the percent contributions is the sum of the atomic orbital coefficient squares. As shown in Figure 4, the highest occupied orbital is composed of three parts with a predominant contribution from the phenyl ring. The LUMO and some lower-energy occupied MOs (HOMO-1 and $\mathrm{HOMO}-2$ ) have a predominant contribution from the pyrene ring. The HOMO-LUMO band gap obtained from the MO calculations was $3.42 \mathrm{eV}$.

Theoretical Electronic Absorption Spectra. TD-DFT computations were performed to predict the electronic transition energies and intensities of the 10 lowest-energy singlet transitions of PyDMA. Selected low-lying singlet excited states together with their excitation energies and oscillator strengths assigned for PyDMA are displayed in Table 2. An experimental model for an excited state corresponds to the excitation of an electron from an occupied to a virtual molecular orbital (i.e., a one-electron picture). However, the excited states calculated herein demonstrate that excited-state electronic structures are best described in terms of multiconfigurations, wherein a linear combination of several occupied-to-virtual MO excitations comprises a given optical transition. Each excited state is assigned based on the compositions of the occupied and virtual MOs of the dominant configuration(s). The theoretical and experimental UV-vis spectrum in solid along with the electron density difference maps (EDDMs) assigned to each transition is shown in Figure 3. It depicts the electron density changes between the ground and excited states upon different electronic excitations. Visualization of these difference density plots allows one to 


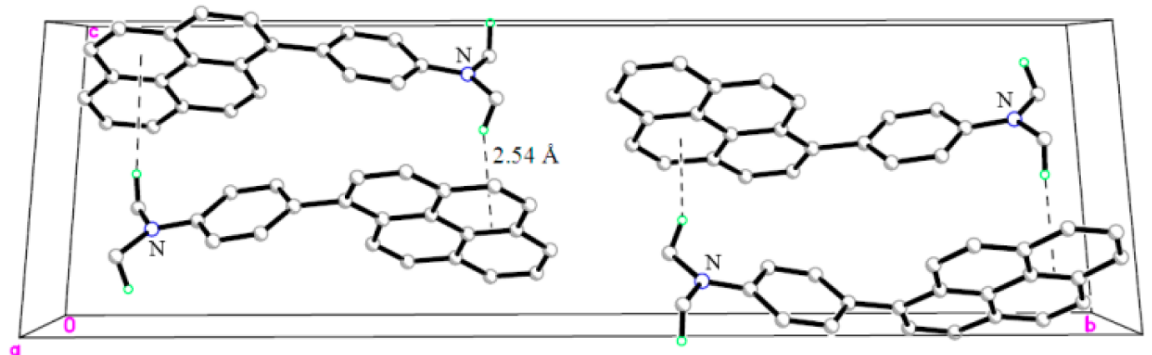

Figure 2. Packing of PyDMA showing extended chain along the $b$-axis by the intermolecular $\mathrm{C}-\mathrm{H} \cdots \pi$ interactions (dashed lines).

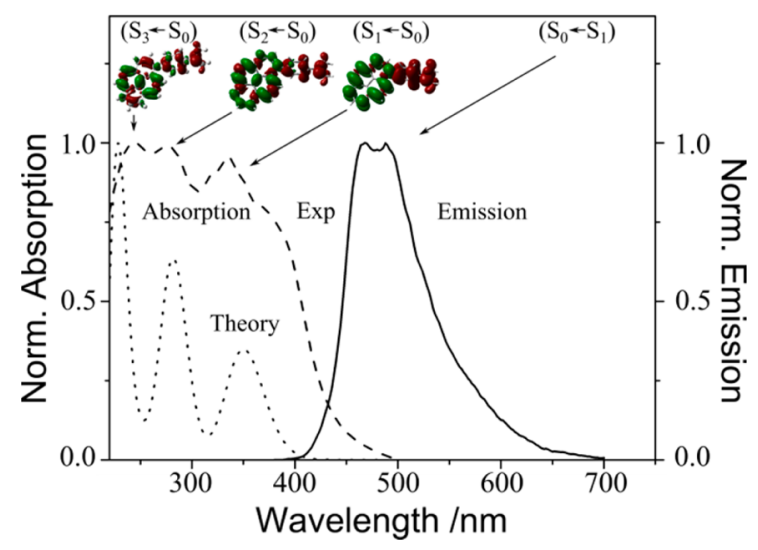

Figure 3. Theoretical and experimental UV-vis spectra of PyDMA with electron density difference maps (EDDMs) assigned to each transition. Left: computational prediction and UV-vis absorption. Right: emission spectra.

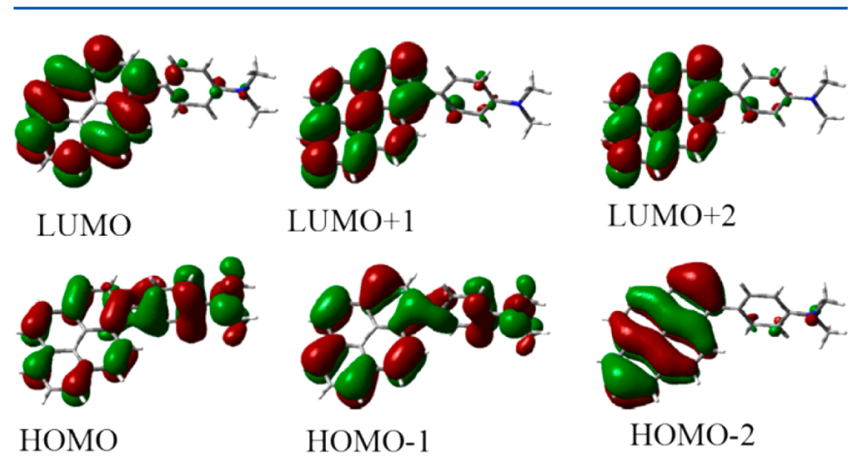

Figure 4. Some of the frontier molecular orbitals of PyDMA.

more readily determine the changes to the electronic features on the excited-state surface and can provide insight into the subsequent geometric changes occurring on the excited-state potential energy surface ${ }^{37,38}$ as well as aid in determining what type of excitation is involved.

Transient Absorption Measurement. The transient absorption spectra measured for PyDMA single crystals are shown in Figure 6a,b. They were measured in the spectral region of $420-750 \mathrm{~nm}$ with $400 \mathrm{~nm}$ excitation. The entire transient spectra exhibit a rise with distinct features as shown in Figures $7 \mathrm{a}$ and $8 \mathrm{a}$. The initial absorption arises from the longer wavelength region $(650-720 \mathrm{~nm})$ along with stimulated emission (450-490 nm), and then a sudden rise in the absorption toward the shorter wavelength side can be seen (Figure 6) that broadens later toward the longer wavelength side. The absence of a negative transient signal of the groundstate bleaching (as typical for PyDMA with a fluorescence quantum yield $>80 \%$ also in solid) might be due to a large
Table 1. G03/B3LYP Calculated One-Electron Energy and Percentage Composition of Selected Frontier MOs of PyDMA

\begin{tabular}{|c|c|c|c|c|c|}
\hline MO & $\begin{array}{c}\text { energy } \\
(\mathrm{eV})\end{array}$ & character & $\begin{array}{c}\text { DMA } \\
(\%)\end{array}$ & $\begin{array}{c}\text { phenyl } \\
(\%)\end{array}$ & $\begin{array}{c}\text { pyrene } \\
(\%)\end{array}$ \\
\hline $90(\mathrm{~V})$ & 0.35 & $\begin{array}{c}\text { phenyl }+ \\
\text { pyrene }\end{array}$ & 1 & 70 & 29 \\
\hline $89(\mathrm{~V})$ & 0.12 & $\begin{array}{r}\text { pyrene }+ \\
\text { phenyl }\end{array}$ & 1 & 30 & 69 \\
\hline $88(\mathrm{~V})$ & -0.35 & $\begin{array}{r}\text { pyrene }+ \\
\text { phenyl }\end{array}$ & 1 & 32 & 67 \\
\hline $87(\mathrm{~V})$ & -0.81 & pyrene & 0 & 3 & 97 \\
\hline $86(\text { LUMO })^{a}$ & -1.23 & pyrene & 0 & 3 & 97 \\
\hline $85(\text { HOMO })^{a}$ & -4.72 & mixed & 28 & 38 & 34 \\
\hline $84(\mathrm{O})$ & -5.18 & pyrene & 15 & 15 & 70 \\
\hline $83(\mathrm{O})$ & -5.95 & pyrene & 1 & 2 & 98 \\
\hline $82(\mathrm{O})$ & -6.50 & phenyl & 0 & 91 & 9 \\
\hline $81(\mathrm{O})$ & -6.61 & pyrene & 1 & 1 & 98 \\
\hline
\end{tabular}

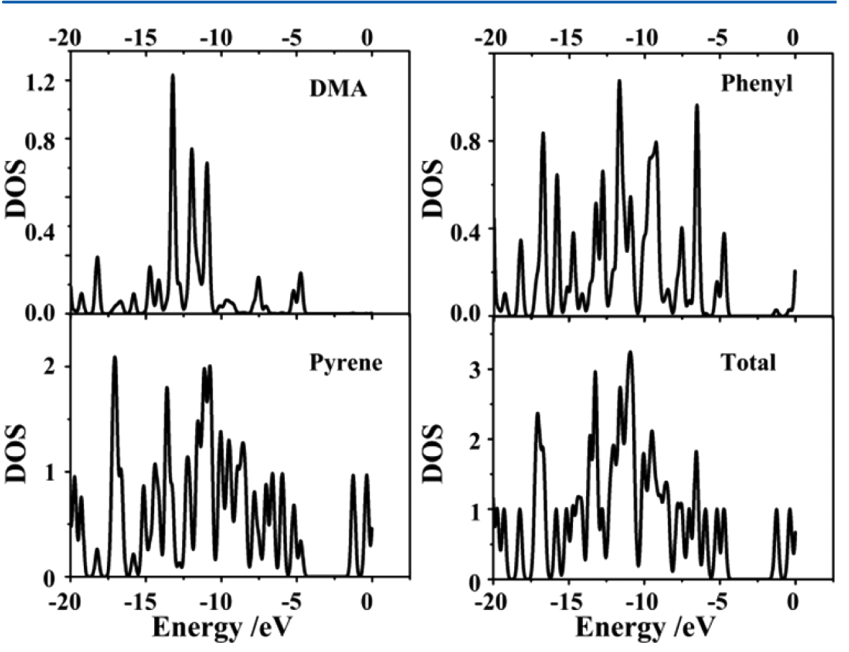

Figure 5. Density of states (DOS) plot of PyDMA.

molar extinction coefficient of the singlet-singlet absorption $\left(S_{1} \rightarrow S_{N}\right)$ in the transient absorption spectra. The transient absorption spectra of PyDMA single crystal has been measured between -20 and 150 ps in steps of $500 \mathrm{fs}$, and only selected time points have been plotted in Figure 6 . The broad transient peaks $\sim 10$ ps from 470 to $550 \mathrm{~nm}$ with small shoulders in the peaks are probably due to the radical cation of DMA/triplet DMA or pyrene triplet state. These small shoulders might presumably be the vibronic features of the radical cation. 39,40 This radical ion formation also indicates the creation of donorto-acceptor charge-transfer state immediately after the excitation which slowly starts broadening toward the red. The 
Table 2. Selected TD-DFT Calculated Excitation Energies and Compositions of the Low-Lying Singlet Excited States of PyDMA

\begin{tabular}{|c|c|c|c|c|c|}
\hline state & $E(\mathrm{eV}) / \lambda(\mathrm{nm})$ & $f$ & $\lambda_{\exp }$ & $\operatorname{transition}^{a}$ & assignment \\
\hline 1 & $2.86 / 432$ & 0.224 & 343 & HOMO $\rightarrow$ LUMO (94\%) & $\left(\mathrm{n} \rightarrow \pi^{*}\right)\left(\pi \rightarrow \pi^{*}\right)$ \\
\hline 3 & $3.60 / 344$ & 0.299 & 278 & $\mathrm{H}-1 \rightarrow$ LUMO $(67 \%)$, HOMO $\rightarrow$ L+1 $(11 \%)$ & $\left(\pi \rightarrow \pi^{*}\right)$ \\
\hline 9 & $4.47 / 277$ & 0.570 & 242 & $\mathrm{H}-2 \rightarrow$ LUMO (29\%), H-1 $\rightarrow$ L+1 $(23 \%)$ & $\left(\pi \rightarrow \pi^{*}\right)$ \\
\hline
\end{tabular}

${ }^{a} \mathrm{H}$ and $\mathrm{L}$ refer to the highest occupied and lowest unoccupied molecular orbitals, respectively.
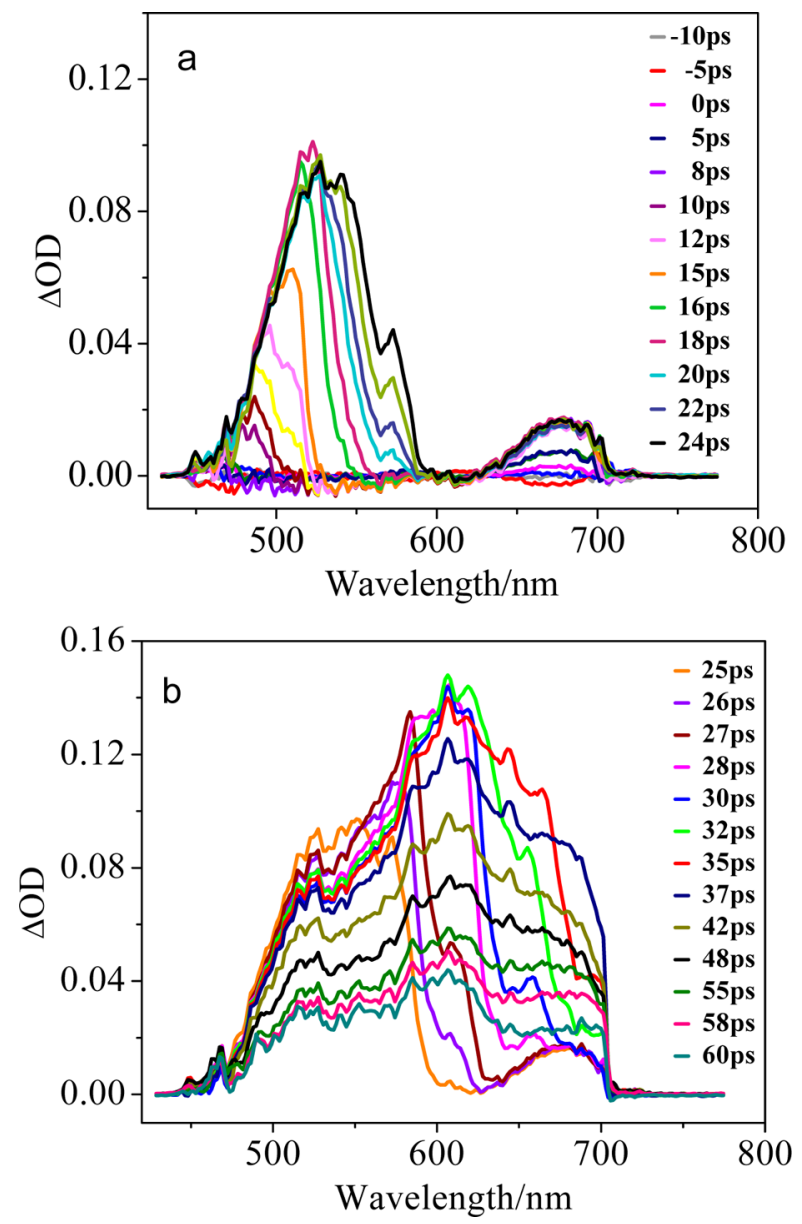

Figure 6. Transient absorption spectra of PyDMA single crystal measured between (a) -10 and 24 ps and (b) 25 and 60 ps (only selected time points are shown).

broad band at around $560 \mathrm{~nm}$ with small humps are due to the $\left(S_{1} \rightarrow S_{N}\right)$ transition of the molecule, and the band at around $650 \mathrm{~nm}$ is due to the $\left(\mathrm{T}_{1} \rightarrow \mathrm{T}_{\mathrm{N}}\right)$ of pyrene moiety. The small humps observed in the whole spectrum might be due to the vibronic or other electronic transitions involved in the system.

In summary, in the first few picoseconds $(\sim 10 \mathrm{ps})$ we could see a broad and less intense transient signal around $650 \mathrm{~nm}$ which is due to the triplet pyrene moiety. After 10 ps emission occurs which we could see as a negative signal around $460 \mathrm{~nm}$, and as time increases it merges with the transient absorption of the radical cation of DMA. After 24 ps we could see a prominent transient band more red-shifted with shoulders, which are due to the singlet transition of the molecule.

For the kinetic interpretation, kinetic traces in the spectral regions of 480-520 nm and 525-690 nm have been depicted. The transient absorption kinetic data was interpreted by fitting to a sum of exponentials convoluted with the Gaussian instrument response function (Figures $7 \mathrm{~b}$ and $8 \mathrm{~b}$ ). The results of these multiexponential fits and of the biexponential fit used

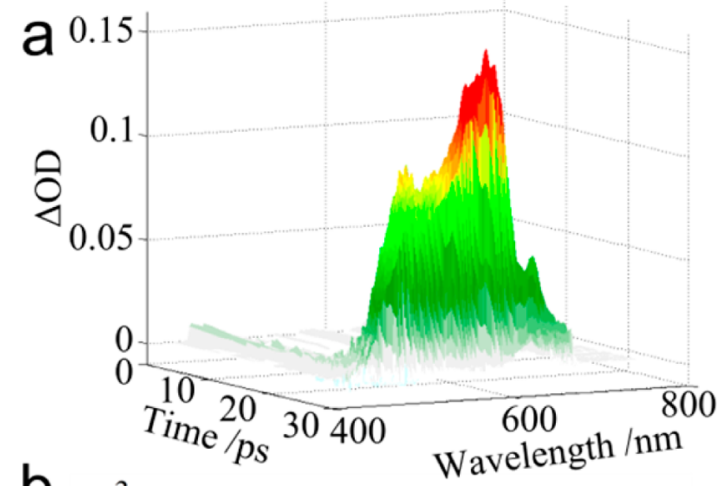

b

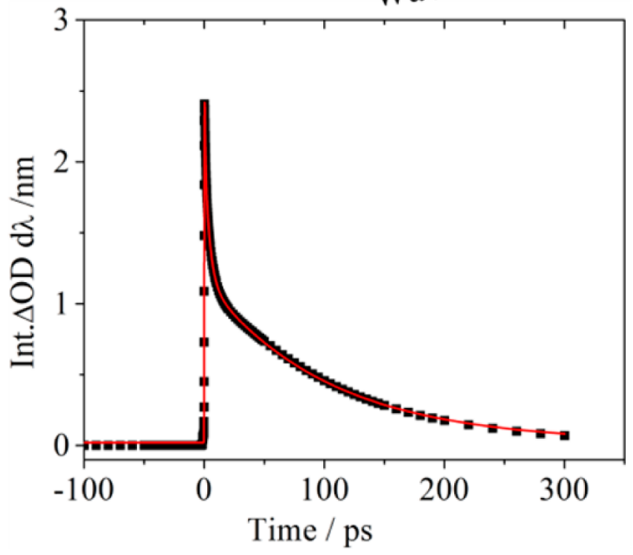

Figure 7. (a)Transient absorption spectra of PyDMA single crystal from early time points $(0-25 \mathrm{ps})$. (b) Transient signal observed as a function of pump-probe delay time at $450-550 \mathrm{~nm}$.

for the time-correlated single photon counting (TCSPC) are summarized in Table 3.

Figure 9 summarizes the kinetic properties listed in Table 3. In the first step, PyDMA is photoexcited with $400 \mathrm{~nm}$ optical pulse light of PyDMA to the excited state of the overlapping $\mathrm{S}_{1} / \mathrm{S}_{2}$ region. Upon excitation the system switches from a molecular conducting to a blocked system (1) because the MOs of DMA moiety lie in a node plane of LUMO (Figure 4). The system relaxes back within 800 fs (2) to the ground state and/or forms a radical ion pair (3). When further probed with optical light (420-720 nm), the system underwent vibrational cooling and enhanced population inversion of the radical ion pair (4). In comparison with the solution state photophysics, in which the rate of electron-transfer process is influenced by external solution interaction, ${ }^{13}$ in solid PyDMA such external effects are negligible or comparable to the influence of liquids with low dielectric permittivity. The molecular diode behavior in solid PyDMA is merely due to the intramolecular electrontransfer process from the donor to acceptor without any dominant environmental influences. 

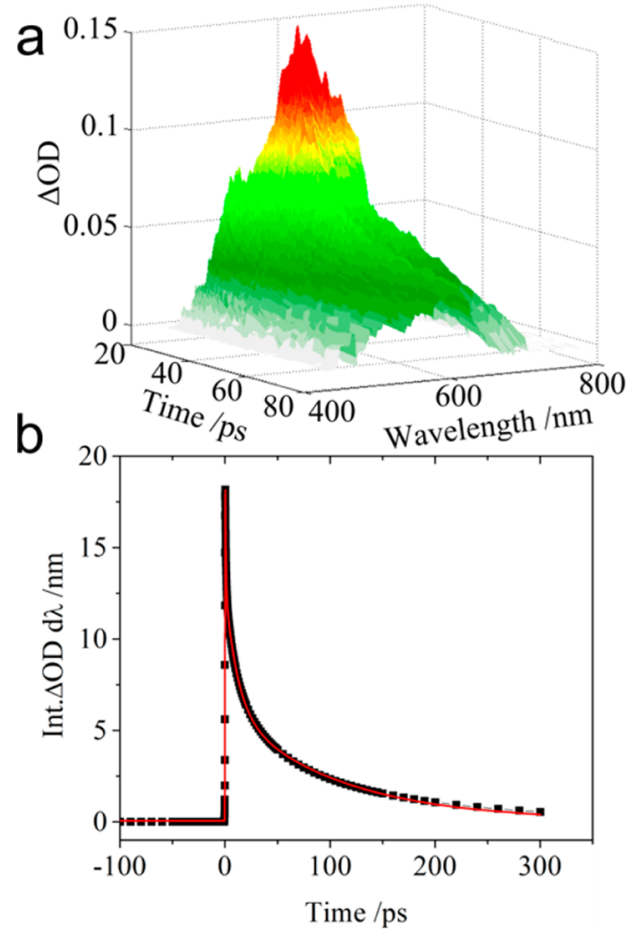

Figure 8. (a)Transient spectra of PyDMA single crystal from early time points $(25-80 \mathrm{ps})$. (b) Transient signal observed as a function of pump-probe delay time at $520-700 \mathrm{~nm}$.

Table 3. Summary of Excited-State Lifetime Data of PyDMA using Levenberg-Marquardt Fit

\begin{tabular}{lll} 
& \multicolumn{2}{c}{ transient lifetime $(\mathrm{ps})$} \\
\cline { 2 - 3 }$\tau_{1}$ & $480-520 \mathrm{~nm}$ & $525-690 \mathrm{~nm}$ \\
$\mathrm{~A}_{1}$ & 0.96 & 0.64 \\
$\tau_{2}$ & $0.52(5.5 \%)$ & $0.97(9.7 \%)$ \\
$\mathrm{A}_{2}$ & 4.20 & 11.58 \\
$\tau_{3}$ & $8.5(90 \%)$ & $6.67(67 \%)$ \\
$\mathrm{A}_{3}$ & 102.86 & 104.34 \\
& $0.42(4.4 \%)$ & $2.27(22.9 \%)$ \\
$\tau_{1}$ & singlet lifetime by SPC \\
$\mathrm{A}_{1}$ & $2.162 \mathrm{~ns}$ & \\
$\tau_{2}$ & $83.25 \%$ & \\
$\mathrm{~A}_{2}$ & $6.223 \mathrm{~ns}$ & \\
\hline
\end{tabular}

\section{CONCLUSIONS}

In summary, we have studied the excited-state dynamics of PyDMA single crystal using femtosecond pump-probe spectroscopy. Beyond what has been employed in transient absorption spectroscopy, we use the crystal structure ${ }^{36}$ for predicting optical properties of PyDMA in order to give a more precise interpretation of the femtosecond transient absorption spectroscopy. The reaction dynamics of molecular diode allowed a detailed description of the electronic property changes during optical excitation. For this system it is not essential that the electron $\mathrm{D}-\mathrm{A}$ system moves or tilts against each other. As one could see on the HOMO-LUMO description, a pure optical transition switches the system from conducting to a blocked system because the MOs of DMA moiety lies in a node plane of the LUMO. Within $800 \mathrm{fs}$ the system relaxes back to the ground state and/or forms a radical ion pair. This two-pathway decay property is the

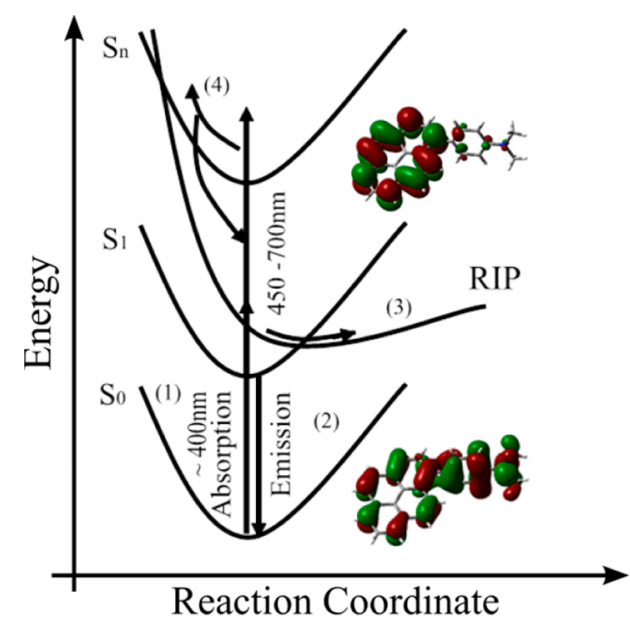

Figure 9. Reaction dynamics of molecular diode PyDMA. (1) Switching from conducting to a blocked system. (2) Stimulated emission. (3) Formation of radical ion pair. (4) Vibrational cooling and enhanced population inversion of the radical ion pair.

surprising results of our study for this system. The observation of an additional radical ion pair formation in solid state has to be taken into account.

\section{ASSOCIATED CONTENT}

\section{S Supporting Information}

X-ray crystallographic information (CIF, CCDC 963294) for the title compound. This material is available free of charge via the Internet at http://pubs.acs.org.

\section{AUTHOR INFORMATION}

\section{Corresponding Authors}

*E-mail: sreevidya.thekku.veedu@desy.de.

*E-mail: simone.techert@desy.de.

\section{Author Contributions}

S.T.V. grew the crystals; performed the experiment; treated, analyzed, and evaluated the experimental data; and wrote the manuscript. D.R., M.S., and R.K. contributed equally to the work. They provided their expertise in data evaluation and critical comments. S.T. supervised the work.

Notes

The authors declare no competing financial interest.

\section{ACKNOWLEDGMENTS}

S.T. thanks DFG, SFB 755 and SFB 1073 for financial support. S.T.V. thanks G. Busse and L. Busse for their technical help and also Z. Yin for his valuable help.

\section{REFERENCES}

(1) Dapprich, J.; Walter, N.; Salinque, F.; Staerk, H. Base-Dependent Pyrene Fluorescence Used for In-Solution Detection of Nucleic Acids. In Proceedings of the 4th International Conference on Methods and Applications of Fluorescence Spectroscopy; Birch, D., Miller, J., Eds.; 1997, 7, 87S-89S.

(2) Soujanya, T.; Philippon, A.; Leroy, S.; Vallier, M.; Fages, F. Tunable Photophysical Properties of Two 2,2'-Bipyridine-Substituted Pyrene Derivatives. J. Phys. Chem. A 2000, 104, 9408-9414.

(3) Simon, J. A.; Curry, S. L.; Schmehl, R. H.; Schatz, T. R.; Piotrowiak, P.; Jin, X.; Thummel, R. P. Intramolecular Electronic Energy Transfer in Ruthenium(II) Diimine Donor/Pyrene Acceptor Complexes Linked by a Single C-C Bond. J. Am. Chem. Soc. 1997, 119, 11012-11022. 
(4) Techert, S.; Schmatz, S.; Wiessner, A.; Staerk, H. Photophysical Characteristics of Directly Linked Pyrene-Dimethylaniline Derivatives. J. Phys. Chem. A 2000, 104, 5700-5710.

(5) Onkelinx, A.; Schweitzer, G.; De Schryver, F. C.; Miyasaka, H.; Van der Auweraer, M.; Asahi, T.; Masuhara, H.; Fukumura, H.; Yashima, A.; Iwai, K. Femto- to Microsecond Excited State Relaxation of 9-(4-(N,N-Dimethylamino)phenyl)phenthrene and 4-(9-Phenathryl)-3,5-N,N-tetramethylaniline. J. Phys. Chem. A 1997, 101, 50545062 .

(6) Ramos, A. S. F.; Techert, S. A Directly Linked Pyrenedimethylaniline Derivative as a Potential Biochemical Sensor for the Microenvironmental Dielectric Properties of the Active Site of Enzymes. Phys. Chem. Chem. Phys. 2003, 5, 5176-5181.

(7) Chen, L. X.; Xiao, S.; Yu, L. Dynamics of Photoinduced Electron Transfer in a Molecular Donor-Acceptor Quartet. J. Phys. Chem. 2006, 110, 11730-11738.

(8) Wasielewski, M. R. Photoinduced Electron Transfer in Supramolecular Systems for Artificial Photosynthesis. Chem. Rev. 1992, 92, 435-461.

(9) Willemse, R. J.; Piet, J. J.; Warman, J. M.; Hartl, F.; Verhoeven, J. W.; Brouwer, A. M. Stepwise versus Direct Long-Range Charge Separation in Molecular Traids. J. Am. Chem. Soc. 2000, 122, 37213730.

(10) Kuzyk, M. G. Using Fundamental Principles to Understand and Optimize Nonlinear-optical Materials. J. Mater. Chem. 2009, 19, 7444-7465.

(11) May, J. C.; Biaggio, I.; Bureš, F.; Diederich, F. Extended Conjugation and Donor-acceptor Substitution to Improve the Thirdorder Optical Nonlinearity of Small Molecules. Appl. Phys. Lett. 2007, 90, 251106-1-3.

(12) Bureš, F.; Pytelaa, O.; Kivalab, M.; Diederichb, F. Solvatochromism as an Efficient Tool to Study $N, N$-dimethylaminoand cyano- Substituted $\pi$-conjugated Molecules with an Intramolecular Charge-transfer Absorption. J. Phys. Org. Chem. 2011, 24, 274-281.

(13) Techert, S.; Schmatz, S.; Wiessner, A.; Staerk, H. Time-resolved Fluorescence and Solvatochromy of Directly Linked Pyrene-DMA Derivatives in Alcoholic Solution. J. Phys. Chem. B 2001, 105, 75797585.

(14) Marcus, R. A. Electron Transfer Reactons in Chemistry. Theory and Experiment. Rev. Mod. Phys. 1993, 65, 599-610.

(15) Dewar, M. J. S.; Mole, T. Palladised Charcoal as a Catalyst for the Reduction of Aromatic Nitro-Compounds by Hydrazine Hydrate. J. Chem. Soc. 1956, 7, 2556-2557.

(16) Norman, R. O. C.; Thompson, G. A.; Waters, W. A. The Reactions of Pyrene with Free Radicals and with Sodium. J. Chem. Soc. 1958, 12, 175-179.

(17) More, R.; Busse, G.; Hallmann, J.; Paulmann, C.; Scholz, M.; Techert, S. Photodimerization of Crystalline 9-Anthracene Carboxylic Acid: A Nontopotactic Autocatalytic Transformation. J. Phys. Chem. C 2010, 114, 4142-4148.

(18) Kabsch, W. Automatic Processing of Rotation Diffraction Data from Crystals of Initially Unknown Symmetry and Cell Constants. J. Appl. Crystallogr. 1993, 26, 795-800.

(19) Sheldrick, G. M. A Short History of SHELX. Acta Crystallogr. 2008, A64, 112-122.

(20) Spek, A. L. Structure Validation in Chemical Crystallography. Acta Crystallogr. 2009, D65, 148-155.

(21) Ramos, A.; Techert, S. Influence of the Water Structure on the Acetylcholinesterase Efficiency. Biophys. J. 2005, 89, 1990-2003.

(22) Michael W. PicoQuant GmbH, Technical Note. TCSPC v.2.1. 2009, 1.

(23) Becke, A. D. Density-functional Thermochemistry. III. The Role of Exact Exchange. J. Chem. Phys. 1993, 98, 5648-5652.

(24) Lee, C.; Yang, W.; Parr, R. G. Development of the Colle-Salvetti Correlation-energy Formula into a Functional of the Electron Density. Phys. Rev. B. 1988, 37, 785-789.

(25) Frisch, M. J.; Trucks, G. W.; Schlegel, H. B.; Scuseria, G. E.; Robb, M. A.; Cheeseman, J. R.; Montgomery, J. A., Jr.; Vreven, T.;
Kudin, K. N.; Burant, J. et al. Gaussian 03, revision B.04; Gaussian, Inc.: Wallingford, CT, 2004.

(26) Barone, V.; Cossi, M. Quantum Calculation of Molecular Energies and Energy Gradients in Solution by a Conductor Solvent Model. J. Phys. Chem. A 1998, 102, 1995-2001.

(27) Cossi, M.; Barone, V. Time-dependent Density Functional Theory for Molecules in Liquid Solutions. J. Chem. Phys. 2001, 115, 4708-4717.

(28) Cossi, M.; Rega, N.; Scalmani, G.; Barone, V. Energies, Structures, and Electronic Properties of Molecules in Solution with the C-PCM Solvation Model. J. Comput. Chem. 2003, 24, 669-681.

(29) Casida, M. E.; Jamorski, C.; Casida, K. C.; Salahub, D. R. Molecular Excitation Energies to High-lying Bound States from Timedependent Density-functional Response Theory: Characterization and Correction of the Time-dependent Local Density Approximation Ionization Threshold. J. Chem. Phys. 1998, 108, 4439-4449.

(30) Debnarova, A.; Techert, S.; Schmatz, S. Ab Initio Treatment of Time-resolved X-ray Scattering: Application to the Photodimerization of Stilbene. J. Chem. Phys. 2006, 125, 224101-1-9.

(31) Browne, W. R.; O’Boyle, N. M.; McGarvey, J. J.; Vos, J. G. Elucidating Excited State Electronic Structure and Intercomponent Interactions in Multicomponent and Supramolecular Systems. Chem. Soc. Rev. 2005, 34, 641-663.

(32) O’Boyle, N. M.; Vos, J. G. Software News and Updates cclib: A Library for Package-Independent Computational Chemistry Algorithms GaussSum 2.2. J. Comput. Chem. 2008, 29, 839-845.

(33) Operator's Manual: VerdiTM Diode-Pumped Laser; Coherent Inc.: Santa Clara, CA, 1997.

(34) Operator's Manual: The Coherent Mira Optima 900-F Laser; Coherent Inc.: Santa Clara, CA, 2004.

(35) Hurricane Users' Manual, 1st ed.; Spectra-Physics Lasers: Mountain View, CA, 2000.

(36) Thekku Veedu, S.; Scholz, M.; Kia, R.; Paulmann, C.; Techert, S. N,N-Dimethyl-4-(pyren-1-yl)aniline. Acta Crystallogr. 2014, E70, O16.

(37) Burdzinski, G.; Gustafson, T. L.; Hackett, J. C.; Hadad, C. M.; Platz, M. S. The Direct Detection of an Aryl Azide Excited State: An Ultrafast Study of the Photochemistry of para-and ortho-Biphenyl Azide. J. Am. Chem. Soc. 2005, 127, 13764-13765.

(38) Wiberg, K. B.; Hadad, C. M.; Breneman, C. M.; Laidig, K. E.; Murcko, M. A.; LePage, T. J. The Response of Electrons to Structural Changes. Science 1991, 252, 1266-1272.

(39) Bree, A.; Vilkos, V. V. B. A Study of Some Singlet and Triplet Electronic States of Pyrene. Spectrochim. Acta 1971, 27A, 2333-2354.

(40) Vala, M.; Szczepanski, J.; Pauzat, F.; Parisel, O.; Talbi, D.; Ellinger, Y. Electronic and Vibrational Spectra of Matrix-Isolated Pyrene Radical Cations: Theoretical and Experimental Aspects. J. Phys. Chem. 1994, 98, 9187-9196. 